
use of the Martinez Pinyon (Pinus maximartinezii Rzed.) in Juchipila, Zacatecas, Mexico

\title{
Elementos para la gestión de la conservación, manejo y aprovechamiento del Pino Azul (Pinus maximartinezii Rzed.) en Juchipila, Zacatecas, México
}

\author{
Daniel Hernández-Ramírez ${ }^{1 *}$, Eduardo Alanís-Rodríguez¹, \\ Martí Boada ${ }^{2}$, Carles Barriocanal2 ${ }^{2}$, Roser Maneja ${ }^{2}$ y Jorge Bluhm-Gutiérrez ${ }^{3}$
}

\section{Resumen}

El manejo, uso y conservación de los recursos naturales se ha venido realizado sin considerar algunos importantes factores sociales. Concretamente el caso del pino azul (Pinus maximartinezii Rzed.) en Zacatecas, se propone que los poseedores de predios con esta especie nativa, deban ser los responsables directos de conservarlo. En el presente artículo se delimitan las bases para que sean ellos mismos los actores principales para llevar a cabo la conservación, uso y aprovechamiento de pino azul en Juchipila, Zacatecas; siendo que es una especie catalogada como microendémica y sujeta a protección especial por la NOM-059-SEMARNAT-2010 para México y por la Union International Conservation Nature (UICN) como en Peligro (EN); siendo la Comisión Nacional de Áreas Naturales Protegidas (CONANP) la instancia que con el respaldo del Plan de Conservación y Manejo del Área Natural Protegida la que procura llevar a cabo acciones de manejo y conservación en la Cuenca Alimentadora del Distrito Nacional de Riego 043 (CADNR 043), específicamente en la subcuenca del Río Juchipila y en vinculación con los propietarios de predios con pino azul.

Palabras clave: Propietarios. Manejo. Conservación. Restauración. Plan de conservación y manejo.

\footnotetext{
${ }^{1}$ Instituto Regional del Patrimonio Mundial en Zacatecas-Secretaría del Agua y Medio Ambiente (IRPMZ-SAMA). Dirección: Juan de Tolosa No. 831, Centro Histórico, Zacatecas, Zac. México

${ }^{2}$ Instituto de Ciencias y Tecnologías Ambientales de la Universidad Autónoma de Barcelona. ICTA-ICP, Edificio Z Campus de la UAB 08193 Bellaterra (Cerdanyola del Vallès) Barcelona. Tel. (+34) 935868777 | Fax. (+34) 935814070

${ }^{3}$ Unidad Académica de Ciencias de la Tierra, Universidad Autónoma de Zacatecas (UAZ). Zacatecas, Zacatecas, México. Avenida Universidad 108, Progreso, 98050 Zacatecas, Zac.

* E-mail: dhernan87@hotmail.com, móvil +52 149214301 31)
} 


\section{Abstract}

Management, use and conservation of natural resources have been done without considering some important social factors. Specifically, in the case of Martinez pinyon (Pinus maximartinezii Rzed.) in Zacatecas, property owners with this native species should be directly responsible for its conservation. This work defines the bases, so they can take the main role in management and better use of "pino azul" at Juchipila, Zacatecas, which has been listed as micro-endemic and subjected to special protection by the Mexican norm NOM-059-SEMARNAT-2010 and the International Union for Conservation of Nature (IUCN) as endangered (EN). The National Commission of Protected Natural Areas (CONANP) is the agency that seeks to perform management and conservation actions with the support of the Conservation and Management Plan of the Protected Natural Area in the Watershed of the National Irrigation District (CADNR 043), specifically in the sub-basin of the Juchipila River and in connection with property owners of Martinez pinyon.

Keywords: Owners. Management. Conservation. Restoration. Conservation and Management Plan.

\section{Introducción}

La falta de una visión integral en el manejo, uso y conservación de los recursos naturales ha hecho que se tenga una grave carencia en el proceder a todos los niveles de gobierno en México, pues por una parte el manejo se establece en función a un objetivo planteado, este puede ser éticofilosófico, por otra parte se sabe también que existe un interés científico-académico en ciertas áreas, además de los propósitos del ámbito económico propios de todo proyecto de gestión. En ambas situaciones, existen implícitos elementos ambientales, económicos y socioculturales.

En México, las Cuencas Alimentadoras de los Distritos Nacionales de Riego (CADNR) pudieran hacer esta función, sin embargo, se llevan a cabo acciones de conservación controvertidas y en ocasiones inadecuadas (Mittermeier y Mittermeier, 1992).

El municipio de Juchipila, Zacatecas, México (Figura 1) forma parte desde 1949 del área de Protección Forestal y de Repoblamiento de las Cuencas de Alimentación de las Obras de Irrigación de los Distritos Nacionales de Riego, con el objetivo de mantener una veda total e indefinida de la 
tala de árboles en los montes ubicados dentro de las cuencas (DOF, 1949).

Posteriormente, en 2002 se recategorizó y se le nombra Área de Protección de Recursos Naturales (APRN), la cual busca facilitar esquemas de conservación por medio de la permanencia de los servicios ambientales, tales como la precipitación y la abundancia de agua en los sistemas hidrológicos de las cuencas hidrográficas (CONANP, 2014).

El APRN de la CADNR 043, tiene una extensión de más de dos millones de hectáreas (CONANP, 2014). Y existe gran variedad de flora y fauna. Cabe señalar por su situación y singularidad nacional e internacional al Pinus maximartinezii Rzd. o pino azul; es una especie Endémica (E) y sujeta a Protección Especial (Pr) en la NOM-059 (González-Elizondo et al., 2011; López-Mata, 2013; López-Mata y Galván, 2011; SEMARNAT, 2010).

Por su parte, la IUCN incluye al pino azul (Pinus maximartinezii Rzed.), en el grupo de especies en Peligro (EN), su distribución natural es reducida, el hábitat es fragmentado y en franco proceso de deterioro por actividades agrícolas y ganaderas; así mismo, la cantidad de individuos presentes son muy pocos y no cuantificados (Farjon, 2013).

Trabajos anteriores, señalan a la especie con una distribución no homogénea en un espacio de 2,850 ha, las cuales compactadas ocupan 61.59 ha (Ruiz-Garduño et al., 2011).

Estos elementos exhiben una necesidad de estrategias para la sobrevivencia de la especie y su ecosistema (Fernández-Candelas, com. pers.), se requieren mecanismos para que mejore el involucramiento de los propietarios de predios en la protección, conservación, uso, conocimiento y rehabilitación de la zona de distribución natural (Ericson, 2006; Jones-Walters y Cil, 2011; Méndez-López et al., 2014; Otero et al., 2011; Pietrzyk-Kaszynska et al., 2012; Rodríguez-Martínez, 2007; SEMARNAT, 2010; Tole, 2010).

Por su parte, la Comisión Nacional de Áreas Naturales Protegidas (CONANP) es la autoridad mexicana encargada de mantener la representatividad de los ecosistemas mediante la conservación y el manejo sustentable, además de fomentar actividades productivas con criterios de inclusión, equidad y reducción de pobreza (Cámara de Diputados, 2014).

Los Programas de Conservación y Manejo son el instrumento rector de planeación y regulación que establecen actividades, acciones y lineamientos de operación en áreas naturales protegidas (Cámara de Diputados, 2014).

Es así como con base a las carencias con las que actualmente opera el ANP, en donde está esta 
especie de pino azul, se plantea examinar opiniones, actitudes y semejanzas entre la estructura del Plan de Conservación y Manejo y sus líneas estratégicas (Gráfico 1), frente a las perspectivas y nivel de información de los propietarios de la zona de distribución natural de pino (Pinus maximartinezii Rdz.) en el Área de Protección de Recursos Naturales Cuenca Alimentadora del Distrito Nacional de Riego 043, Subcuenca del Río Juchipila, en Zacatecas, con el objetivo de hacer aportaciones puntuales al plan de conservación y manejo.

\section{Materiales y métodos}

La zona de estudio se encuentra en la provincia Sierra Madre Occidental y en la subprovincia fisiográfica Sierras y Valles Zacatecanos y forma parte de la cordillera conocida regionalmente como Sierra de Morones, con altitudes que varían de 1,110 a 2,500 msnm y un clima prevaleciente de semicálido, semiárido a templado (Ruiz-Garduño et al., 2011); está dentro de la Región Hidrológica No. 12, denominada como Lerma- Santiago, en la subcuenca del Río Juchipila al sur del estado de Zacatecas, México; específicamente en el municipio de Juchipila. Su ubicación geográfica se encuentra en las coordenadas extremas $103^{\circ} 12^{\prime}-103^{\circ} 15^{\prime}$ W y $21^{\circ} 19^{\prime}-21^{\circ} 23^{\prime} \mathrm{N}$ (Figura 1 y Figura 2 ).

\subsection{Muestreo: encuesta y colecta de datos}

El estudio se basó en la aplicación de una encuesta dirigida a los propietarios de predios con pino azul, se optó por esta metodología por ser considerada una importante fuente de información rápida y eficaz, se define como el conjunto de procedimientos estandarizados de investigación, mediante el cual se recogen y analizan datos de una muestra representativa de una población o universo (Casas Anguita et al., 2003).

La encuesta es tipo analítica observacional, con combinación de preguntas cerradas de elección múltiple y con abanico de respuestas. Se buscó obtener información de hechos concretos, intenciones, opiniones, nivel de información, actividades, aspiraciones, motivos y razones (Casas Anguita et al., 2003; Sierra-Bravo, 1994), esto por parte de propietarios de predios con pino azul.

Se entrevistó a trece de los quince propietarios de predios con distribución natural de pino azul en Zacatecas. Las entrevistas se hicieron en el mes de septiembre de 2014. A los encuestados, se les dio a conocer la intención del estudio como un ejercicio académico y con el compromiso de hacerlo público al concluirlo. 
Por otra parte, y como descripción general del sitio, se debe mencionar el entorno de inseguridad prevaleciente en la zona, lo que pudiera influir en las respuestas, asimismo, dicha situación no es exclusiva de esta zona, se sabe que está extendida a gran parte del territorio mexicano (Carbonell, 2011).

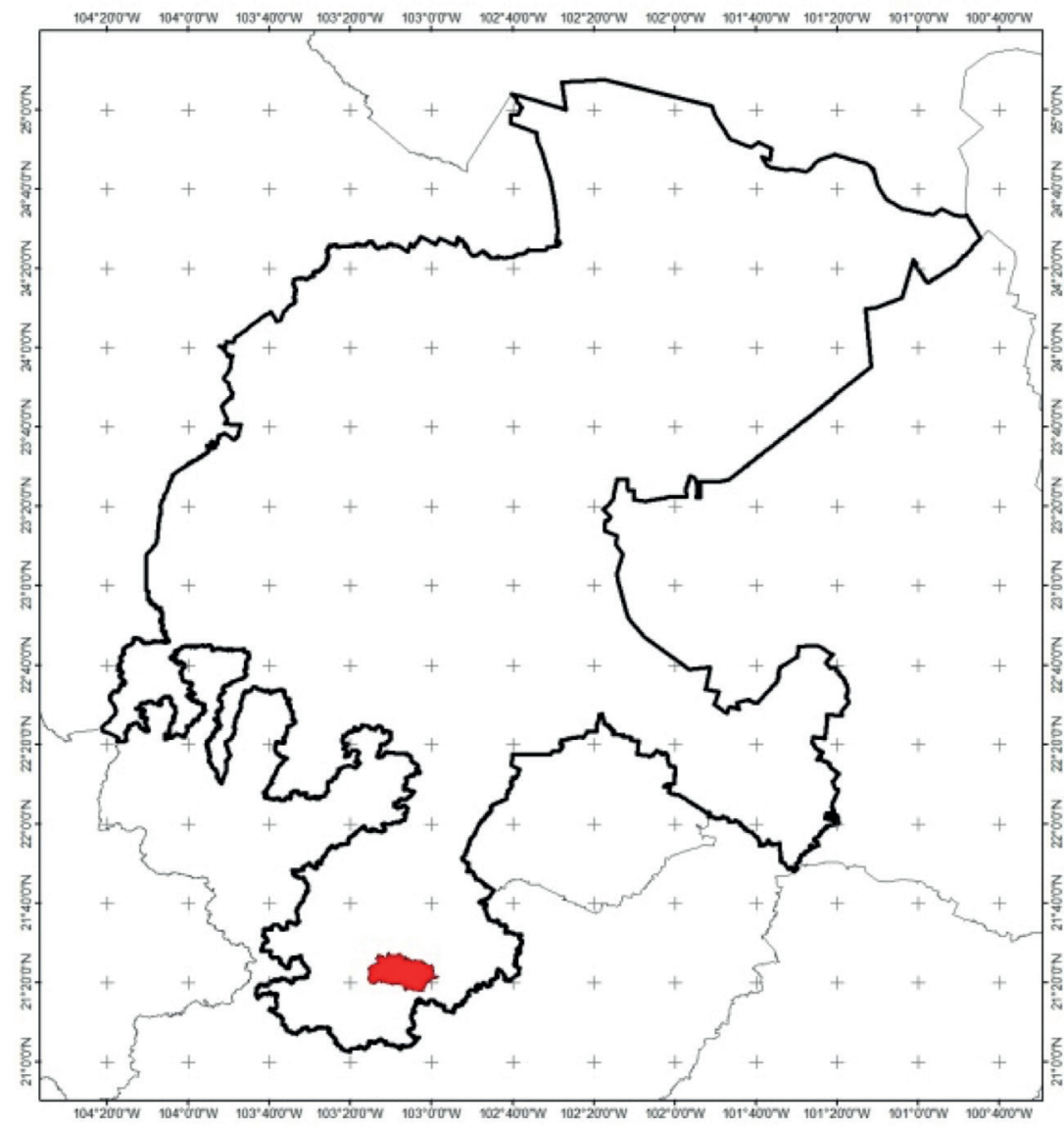

\section{ÁREA DE DISTRIBUCIÓN}

Pino Azul (Pinus maximartinezii)

Cerro de Piñones, Sierra de Morones

Municipio de Juchipila, Zacatecas

\section{Descripción}

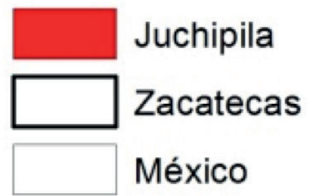

México

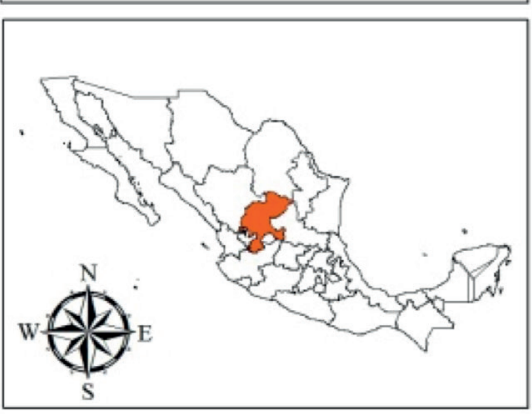

Figura 1. Ubicación del área de estudio (Elaboró: Serra-Ortiz, 2014)

Asimismo, la sistematización del trabajo se hizo con base al esquema que presenta CONANP para la ejecución de los Planes de Conservación y Manejo en cada ANP en territorio nacional (Figura 3).

\section{Resultados y discusión}

Del análisis hecho, once entrevistados (84\%) son del sexo masculino y dos (16\%) del femenino; las edades oscilan entre los cuarenta a los ochenta y tres años; ninguno indicó interés por cambiar de lugar de residencia en los próximos cinco años. Todos los encuestados son pequeños propietarios desde hace más de diez años y la forma de obtener sus predios fue diferenciada: cinco de ellos 
(38\%) lo son por compra-venta directa y ocho (62\%) como beneficiarios de herencias. El hecho de que todos sean pequeños propietarios nos indica que pueden tomar decisiones directas sobre sus predios, y de que deberían tener interés en tenerlos en las mejores condiciones y siendo productivos.

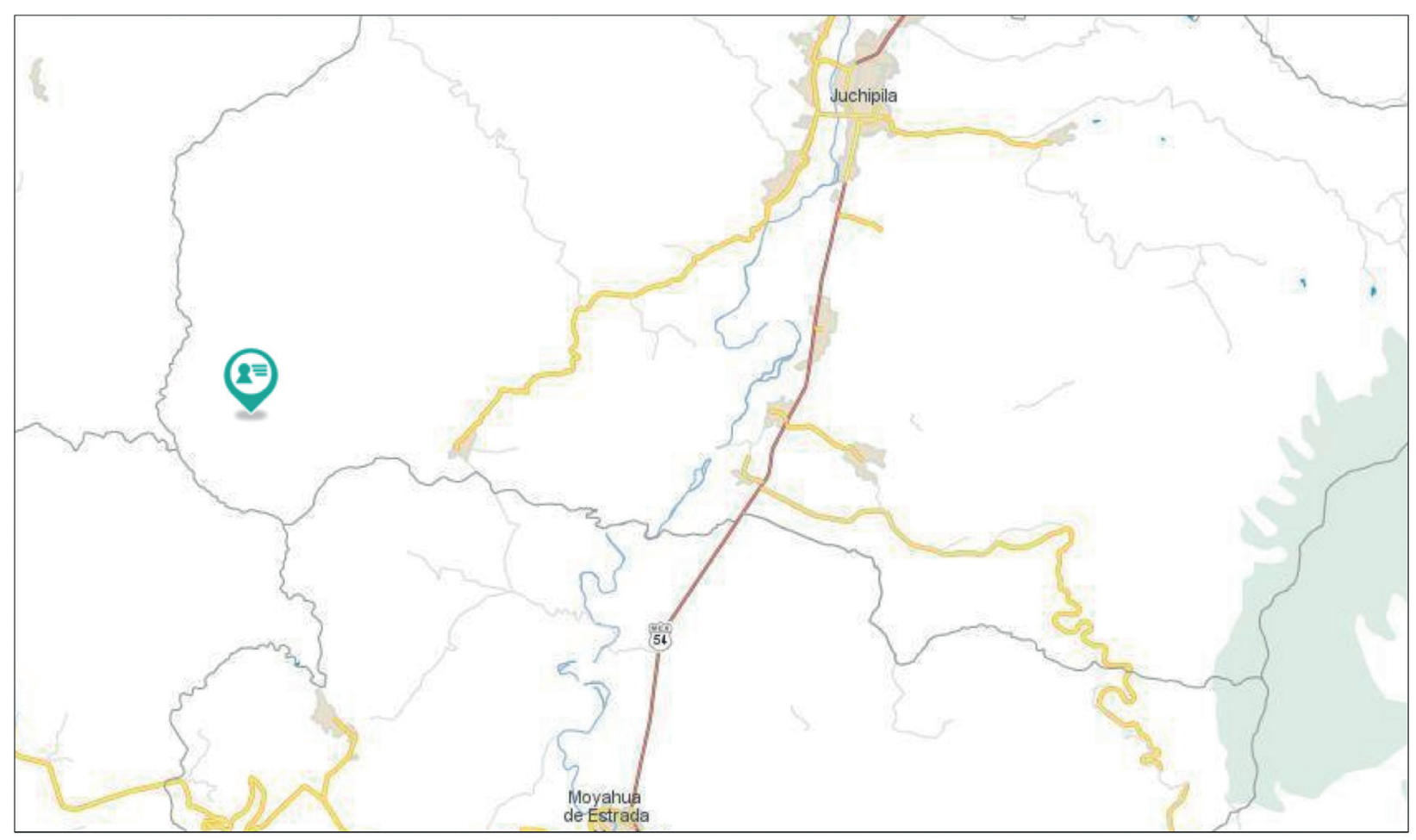

Figura 2. Ubicación del Cerro Piñones, Juchipila, Zacatecas (Fuente: Elaboración propia a partir de Mapa Digital de México, INEGI 2018)

La extensión territorial de cada propietario es variada: va desde las diez hasta las 350 ha (RuizGarduño et al., 2011). La escolaridad también es diferenciada: nueve (70\%) refieren tener educación primaria, dos (15\%) secundaria y dos (15\%) estudios universitarios.

Los ingresos económicos se presentan de la siguiente manera: siete (54\%) basan sus ingresos en la venta de ganado, cuatro (31\%) en actividades agropecuarias y dos (15\%) en la venta de piñón de pino azul y otras actividades complementarias. Se observa que todos los propietarios dependen fundamentalmente de sus tierras y las aprovechan activamente.

Los resultados presentados son en un esquema similar a la estructura de los Planes de Conservación y Manejo para cada área protegida de México; se contemplan dos líneas estratégicas, por un lado, las acciones directas (subprogramas de vigilancia, manejo y restauración) y por otra parte las acciones indirectas (subprogramas de conocimiento, cultura y gestión, Figura 3). 


\subsection{Líneas estratégicas de acción directa}

La necesidad de establecer acciones preventivas y correctivas por parte de la CONANP como órgano regulador, ha traído consigo ciertos aspectos esenciales. En este sentido se encontró que doce propietarios (92\%) de predios con pino azul, desconocen el sentido implícito de la vigilancia ambiental, mientras que un propietario (8\%) expresó conocerlo.

En este sentido doce propietarios (92\%) mencionan la necesidad de una organización grupal para llevar a cabo acciones de vigilancia ambiental, se abre una oportunidad de capacitación específica, dirigida al grupo. Es así que nueve de ellos (70\%) indican que la responsabilidad de vigilar debe estar a cargo de los mismos propietarios de predios con pino azul, cuatro propietarios (30\%) adjudican la responsabilidad a autoridades o policías estatales, federales o municipales. Se tiene pues que la mayoría de los propietarios manifiesta que la responsabilidad de la vigilancia ambiental les corresponde a ellos.

La poca apertura e interés de los propietarios a colaborar en acciones de vigilancia se refleja en que dos personas (15\%) manifestaron acuerdo en participar en grupos organizados para realizar recorridos de vigilancia ambiental, los once restantes (85\%), dijeron no tener disponibilidad para ser capacitados en vigilancia ambiental. En este sentido, el hecho de poder identificar los delitos ambientales recobra una singular importancia en la zona de estudio; de modo general, once propietarios (85\%) dijeron conocer los delitos ambientales y sólo dos (15\%) los desconocen.

De igual forma, seis $(46 \%)$, apuntaron conocer los procedimientos para llevar a cabo una denuncia sobre temas ambientales; siete propietarios (54\%) desconocen el sistema. Este aspecto para ser mejorado requiere la capacitación de los propietarios de los predios en la metodología a seguir para denunciar ante PROFEPA. En cuanto al turismo como componente específico del subprograma de manejo, este incluye a la participación social, entendida como el medio por el cual los habitantes de un área protegida se hacen conscientes de fortalezas y debilidades.

Este componente recibe apoyo de acciones como la educación, la capacitación e interpretación ambiental; en este último componente se encuentra involucrado el turismo, nueve propietarios (69\%) lo perciben en sus predios como una actividad buena, contra cuatro (31\%) como una actividad regular. Es de resaltar que no se presentaron percepciones negativas en cuanto a la actividad turística en los predios. 
Los propietarios de predios con pino azul perciben a los eventuales visitantes en diferentes sentidos: seis (46\%) los han visto realizar actividades de campismo, cinco (38\%) han observado llevan a cabo actividades como caminar y uno (8\%) indica haberlos visto en actividades con vehículos motorizados, otra más $(8 \%)$ menciona actividades no especificadas.

En cuanto al potencial para desarrollar actividades turísticas en la zona de pino azul donde los daños ambientales se reduzcan al mínimo, nueve propietarios (69\%) manifiestan conocer sitios atractivos para visitantes y cuatro (31\%) los desconocen.

Así mismo, ocho propietarios (62\%) conocen lugares arqueológicos o con vestigios de actividades indígenas y diez (77\%) refieren saber de sitios con aguas termales. En cuanto a la circulación de vehículos todo terreno por la zona, once propietarios (92\%) indicaron haber visto vehículos todo terreno en más de una ocasión en sus predios, dos (15\%) comentan haber sufrido destrozos menores en sus predios; el total de propietarios coincide en que la frecuencia de visitas es irregular e inconstante y sólo se les ve en algunos fines de semana de meses específicos.

Once propietarios $(85 \%)$ perciben el paso de vehículos todo terreno como un daño al lugar, razón por la cual rechazan categóricamente recibir turistas o visitantes bajo esas condiciones de movilidad en la zona de pino azul; únicamente dos (15\%), manifiestan deseos de recibirlos con estos vehículos.

Por otra parte, y como elemento de acción directo, la restauración de ecosistemas es un componente esencial para el funcionamiento de las áreas naturales protegidas en México; se busca frenar el deterioro ambiental y recuperar las condiciones originales, siendo requisito fundamental para su éxito, la participación coordinada y efectiva de la comunidad.

Es así como se busca que la integración de propietarios de sitios con distribución natural de pino azul juegue un papel fundamental en la restauración de las áreas protegidas.

En este sentido, diez propietarios (77\%) refieren conocer sitios susceptibles a ser restaurados, tanto con plantas de pino azul como con otras especies. En general, el deterioro ambiental lo perciben como un cambio en la composición de la vegetación por actividades agropecuarias como la ganadería, la agricultura y el aprovechamiento de madera.

Un dato poco claro es el hecho de no aceptar participar en acciones de restauración y de reforestación, nueve propietarios (69\%) se negaron a participar.

Por otra parte, cuatro (31\%) propietarios de predios con presencia de pino azul, dijeron haber 
presenciado la disminución y desaparición de dos especies animales; el lobo mexicano (Canis lupus var. baileyi) y la cotorra serrana (Rhynchopsitta pachyrhyncha).

\section{PROGRAMA DE CONSERVACIÓN Y MANEJO DE ÁREAS NATURALES PROTEGIDAS}

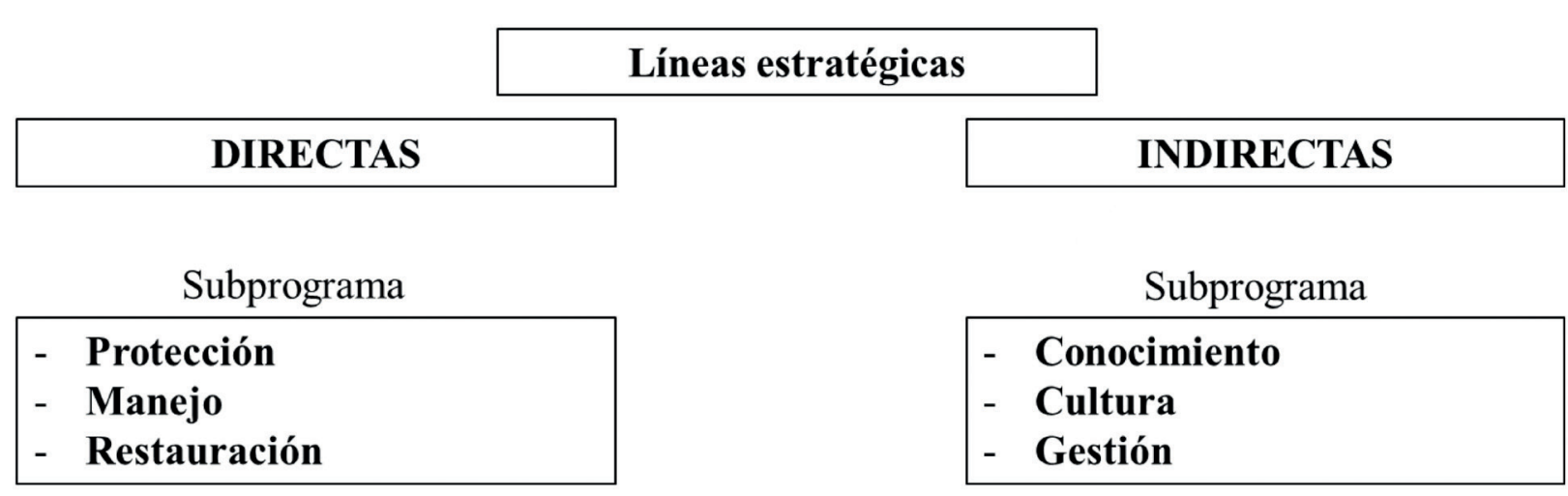

Figura 3. Esquema general de los Planes de Conservación y Manejo, además de las líneas estratégicas y subprogramas que lo componen (Fuente: Elaboración propia a partir de CONANP 2014)

\subsection{Líneas estratégicas de acción indirecta}

El desarrollo de las áreas naturales protegidas está ligado a comprender experiencias empíricas, vinculadas e integradas a conocimientos científicos. Los beneficios necesariamente se deben ver en la consolidación de aspectos como la conservación y la sustentabilidad. Para lograrlo es necesario generar conocimientos de procesos naturales y antropogénicos que se llevan a cabo dentro del área natural protegida.

Reconocer experiencias de los propietarios de predios con pino azul adquiere una importancia relevante; diez de ellos (77\%) reconocen que sus espacios mantienen su cubierta vegetal, no se han dado procesos negativos como incendios forestales, dos de ellos (15\%) mencionan se han tenido cambios en un sentido regular y uno (8\%) refiere cambios drásticos. En base a estos datos se observa que la mayoría de los propietarios procura conservar la vegetación y los suelos en condiciones adecuadas, lo que habla de una conciencia de conservación ambiental.

Doce propietarios (92\%) reconocen el concepto de área protegida o al menos lo han oído mencionar en más de una ocasión, en contraste uno (8\%) lo desconoce en su totalidad. Al preguntar por las ventajas de tener predios dentro de un área protegida, cinco (38\%) manifestaron 
desconocer las ventajas y ocho (62\%) dijeron saberlo de modo parcial. Estas respuestas abren una ventana de oportunidad respecto a la capacitación para mostrar los beneficios que tiene un área natural protegida.

Nueve propietarios de predios (69\%), consideran que el pino azul no es una especie en peligro de extinción, que "en el cerro hay mucho" o que "ese árbol no se acaba, siempre ha estado ahí". Por otra parte, todos los propietarios manifestaron la necesidad de tener capacitaciones técnicas en temas relacionados al pino azul: técnicas de reforestación, procesos de colecta de semilla, comercialización, implementación de viveros forestales y capacitación de huertos de traspatio. Los últimos resultados muestran que los poseedores de los predios desean mejorar su habilitación en temas de gestión ambiental y productiva de sus terrenos.

El componente de educación ambiental es parte del subprograma decultura para la conservación. Se incluyen aspectos como la participación social, como la integración de procesos dirigidos a adquirir conocimientos, al desarrollo de habilidades y a la reorientación de valores y conductas.

Es así como nueve propietarios (69\%) señalan conocer actividades relacionadas con la educación ambiental en su comunidad como diálogos, charlas y sesiones de cine ambiental.

El medio más aceptado por el cual los propietarios del pino azul tienen acercamiento a temas de educación ambiental, es por la televisión, la radio, los periódicos y por conversaciones o diálogos interpersonales. El hecho de considerar importante la educación ambiental genera un clima de apertura e interés por aprender temas novedosos y, en cierta forma a querer compartir experiencias personales de los propietarios de pino azul, qué a pesar de desconocer las razones, consideran importante los elementos que conlleva la educación ambiental.

Haber tenido una experiencia previa con alguna actividad de educación ambiental, les da la oportunidad de interesarse en este tipo de temas y de actividades; once propietarios (85\%) han tenido experiencias previas en charlas, capacitaciones o diálogos relacionados con salud pública (separación de basura y residuos sólidos), con la realización de composta entre otras, sin haber sido abordados temas referentes a conservación o manejo del bosque. Esto es un área de oportunidad, para ofrecerles capacitación en aspectos silvícolas.

Otro elemento importante es la gestión de los recursos naturales, que es el mecanismo por el cual se planifica y determinan políticas, se establecen normas y fomentan actividades, así como los permisos para llevar a cabo algunas actividades. Se busca que la sociedad en general y las 
instituciones en particular, participen de manera ordenada en la conservación del área.

Los elementos de gestión considerados son todas aquellas acciones y políticas que atañen de manera directa a los ecosistemas, a su biodiversidad y a las comunidades asentadas dentro o muy cerca de las áreas protegidas. Es así, como se percibe que la participación y vinculación de instituciones federales, estatales y municipales es poco efectiva, lo que deja entrever aspectos que vulneran elementos ambientales como es el caso de la zona de pino azul.

La carencia de acciones de transversalidad gubernamental, en donde el eje de desarrollo se focalice en el ecosistema de pino azul se ve interrumpido por la falta de coordinación entre los tres órdenes de gobierno, lo que recae en una falta de interés y disposición concretas en las diferentes esferas sociales, económicas y ambientales de atención gubernamental.

Las acciones transversales de coordinación de apoyos y de énfasis de prioridades traerán beneficios a mediano o largo plazo, se contempla que la mitigación de riesgos no será sólo para la espacie de pino azul, sino para todo el ecosistema, que sin duda favorecerá la certidumbre social y económica de la zona.

La regulación de obras y actividades tiene fallos en lo que se refiere a las autorizaciones, ya que se han hecho construcciones de tipo comercial y caminos que evidentemente ponen en riesgo a la especie de pino azul y a todo su ecosistema, se deja en claro que parte del problema es que hay apoyos económicos por parte de algún sector del gobierno, que afecta de manera directa y negativa al ecosistema de pino azul, donde se deja notar en ocasiones una desvinculación y falta de coordinación interinstitucional.

Por otra parte, la carencia de un sistema de señalización en el área natural protegida es otro factor de riesgo al desconocer límites y características del sitio, se pueden efectuar actividades no compatibles con la conservación del área. Por lo antes mencionado, hay muestras de insuficiente actividad relacionada con la implementación de mecanismos de participación social, razón por lo cual el riesgo se incrementa.

En el APRN de la CANDR 043, porción Subcuenca Río Juchipila, donde se encuentra gran parte de la población de pino azul en México, se detectan inconvenientes en la gestión, manejo y conservación de la biodiversidad vinculados con los elementos sociales presentes.

Se observa desvinculación socioecológica, por lo que es imperativo encaminar acciones en beneficio del ecosistema de pino azul en conjunto con los habitantes o usuarios de la zona. Se 
requiere incrementar el conocimiento sobre la compleja relación entre la diversidad cultural y biológica del sistema con el fin de obtener estrategias de conservación exitosas.

El análisis de género de los propietarios deja en desventaja a las mujeres del grupo estudio o poseedoras de predios con pino azul. Se observa una tendencia global donde se les confieren mayores responsabilidades a las mujeres que beneficios en el momento de ser ellas las manejadoras de predios (Aurora-Jonsson, 2011; O’Shaughnessy y Krogman. 2011). Otros estudios indican que el manejo de recursos naturales con la inclusión de mujeres confiere mayores beneficios a la conservación (Meinzen-Dick et al., 1997).

Otro aspecto relevante en el perfil de propietarios de predios con pino azul es el fenómeno de migración hacia los Estados Unidos de Norteamérica, característica distintiva a nivel estatal y nacional (García-Zamora, 2005). Este aspecto permite observar el abandono de tierras de cultivo que en apariencia favorece procesos de regeneración natural del bosque (Padilla, 2006; RuizGarduño et al., 2011).

Los resultados en el apartado de vigilancia muestran la potencialidad de la integración de los dueños de predios con pino azul, donde se tiene gran posibilidad de éxito, ya que los ciudadanos organizados pueden ser actores importantes en la gestión y vigilancia ambiental. Sin embargo, la mayoría de ejemplos de este tipo se han realizado en zonas urbanas (Enqvist et al., 2014), con el argumento que los beneficios que ofrece el cuidado de los ecosistemas van directamente a repercutir de manera favorable en los habitantes de las zonas urbanas.

Uno de los objetivos en que se basa la Procuraduría Federal de Protección al Ambiente (PROFEPA, 2014) en México, es lograr la participación decidida, informada y responsable de los miembros de la sociedad y de sus organizaciones, en la vigilancia, para inducir el cumplimiento de la ley ambiental.

De este modo, la intervención de propietarios se vuelve un factor determinante para el cuidado y la protección del área, fortalecidos con grupos de vigilancia ambiental ya que se desarrollan actividades de cacería no controlada con efectos negativos en el área (Fischer et al., 2014).

Los entrevistados desconocen el concepto de vigilante ambiental, relacionándolo con actividades de policía comunitaria, situación distante del objetivo ambiental; es así como se sitúa en riesgo inminente la población de pino azul y a su entorno, ya que, de presentarse un delito ambiental, los propietarios evitarán seguir un proceso legal por desconocer el procedimiento y 
por temer por su integridad y la de su familia. No obstante, manifiestan interés en participar como grupo organizado.

En relación a la actividad turística en áreas naturales protegidas, se sabe que ha sido un fenómeno global creciente en los últimos 30 años, por ser herramienta para la conservación, e integrar elementos novedosos, por implementar metodologías de evaluación, por ser fuente de ingresos económicos, por la forma de evaluar los daños ocasionados al entorno o simplemente por ser una actividad atractiva y altamente lucrativa (Budowski, 1976; Brightsmith et al. 2008; Giannecchini, 1993; López-Espinosa De los Monteros, 2002; Karanth y DeFries, 2011; Soulé, 1987; Stronza, 2007; Zhou et al., 2013).

En este sentido, es importante considerar al turismo al momento de diseñar políticas de manejo, aprovechamiento y conservación del área natural del pino azul, ya que una decisión incorrecta abriría las puertas a problemas considerables y a amenazas fuertes al ecosistema del pino azul en Zacatecas.

Es así como se percibe una idea arriesgada de la actividad turística en la zona del pino azul, pensada a gran escala y con un alto impacto (Sanyé-Mengual et al., 2014), ya que relacionan turismo con actividades similares a las efectuadas en el centro vacacional en el municipio de San Miguel de Apozol, en Zacatecas, que dista del modelo de ecoturismo.

La propuesta turística para el área de pino azul se debe enfocar a una actividad de bajo impacto ambiental y menor escala, algo parecido al Turismo de Voluntarios (Brightsmith et al., 2008), en donde los visitantes son personas interesadas en participar activa y económicamente en investigaciones científicas, y en que su estancia en el área protegida se restringe a la colaboración en actividades concretas de vigilancia, monitoreo y restauración de ciertas especies, además de integrarse a dinámicas vitales con los habitantes de la zona.

Se ha detectado interés por actividades como el senderismo y el campismo, que pudieran ser complementarias a la actividad de turismo de voluntarios, donde se consideran factores como la regulación de visitantes y un sistema de monitoreo de actividades puntuales, sin dejar de lado un estudio para conocer las aptitudes del sitio (Luque Gil, 2004; Ocaña Ocaña et al., 2012; Ocaña Ocaña et al., 2013) además de ser conducido estrechamente con actividades de educación ambiental.

Se visualiza otro punto de riesgo: el uso de vehículos todo terreno. Estos automóviles dañan seriamente los ecosistemas cuando circulan fuera de las pistas y caminos (Groom et al., 2007; 
Wolcott y Wolcott, 1984). Aun con esto, en el estado de Zacatecas, y en particular en la zona de estudio, se ha incrementado la actividad, con poco o nulo control y regulación.

Los propietarios de predios desconocen los daños a corto, mediano y largo plazo de dichas actividades. Si bien la vigilancia y el uso turístico son importantes, lo es también la formulación de estrategias que ayuden al cumplimiento de los objetivos de conservación como la restauración de áreas naturales protegidas.

Un punto crítico es la participación activa de habitantes y propietarios (Toledo, 2005; Camargo Ponce de León, 2007; Turbay Ceballos et al., 2013) de la zona de distribución natural del pino azul; es así como los propietarios indican conocer sitios susceptibles para llevar a cabo acciones de restauración, no sólo con plantas de pino, sino con otras que saben se pueden desarrollar en conjunto con la especie en particular y que desde la perspectiva de restauración cabe considerar (Turbay Ceballos et al., 2013; Camargo Ponce de León, 2007; Toledo, 2005).

Se observa una idea controvertida del proceso de participación (Karki, 2013; Buta et al., 2014), ya que se percibe la concentración de actividades y de beneficios económicos en un reducido sector de propietarios, con lo que se abandona la idea de algunos autores de manejar la conservación de recursos naturales como una relación de beneficio de ambas partes (Karki, 2013).

En lo referente a restauración y participación de los propietarios de terrenos con pino azul, hay la posibilidad de llevar a cabo acciones en beneficio de la especie y de los habitantes de la zona con programas de restauración y recuperación in situ (Boada et al., 2013; González-Oliva et al., 2011), es decir, programas encaminados a reproducción y reforestación con participación de poblaciones locales, donde se consideran tanto características sociales, como económicas y ambientales. La discusión entre conservar in situ y ex situ, es actual y da lugar a la generación de nuevos conocimientos (Braverman, 2014).

Otro aspecto relativo a la restauración del medio natural corresponde al momento en que se abandonan tierras de cultivo en zonas aledañas al pino azul, este fenómeno tiene relación directa con el incremento de emigración a los Estados Unidos de Norteamérica (Ruiz-Garduño et al., 2011; García-Zamora, 2005) y parece reportar beneficios en el ecosistema, aunque haría falta un estudio en profundidad de este aspecto.

Al respecto de especies de fauna silvestre que hayan visto desaparecer o disminuir sus poblaciones, se menciona a dos: al lobo mexicano (Canis lupus Baileyi) y a la cotorra serrana 
(Rhynchopsitta pachyrhyncha), el analizar a ambas especies ofrece la posibilidad de llevar a cabo actividades de concienciación ambiental con esquemas de participación social, donde las comunidades aledañas a la zona de estudio enriquezcan y compartan su visión con personal interesado en el tema, dando lugar a un intercambio de ideas muy enriquecedor para ambas partes (Beschta y Ripple, 2010).

Para el lobo mexicano (Canis lupus. var. Baileyi), hay acciones que pueden ser tomadas en cuenta con el objetivo de lograr un pago económico a los propietarios de predios con pino azul, por acciones que de manera directa o indirecta beneficien la reintroducción y mantenimiento de individuos de la especie, procurar su reproducción y establecimiento de poblaciones viables genéticamente (Breck et al., 2011). Situación similar es para la especie de cotorra serrana (Rhynchopsitta pachyrhyncha).

El Programa de Acción para la Conservación de la Especie (PACE, 2007) establece que se deben identificar los lugares y las acciones a realizar para la restauración de zonas forestales ocupadas por esta especie. La gran mayoría de los propietarios de pino azul no conocen el concepto de área protegida, lo que ofrece un punto de partida para el intercambio de ideas e intereses, tanto de los propietarios como de los gestores oficiales, ya que los propietarios sólo ven las ventajas de tener predios en el área en función de los beneficios económicos que estos les generen.

Así mismo, el desconocimiento por parte de la mayoría de los propietarios referente al estado de conservación del pino azul en la zona de Juchipila es alarmante. En este sentido, urge implementar estrategias para compartir conocimientos relacionados a la situación global de la abundancia de la especie de pino azul en Zacatecas.

Se pretende hacer un ejercicio de aprendizaje mutuo (Hague et al., 2010), en donde los poseedores de predios con pino se incluyan en procesos de formulación de propuestas y sobre todo en la implementación de actividades dirigidas a la conservación de todo en su conjunto, donde se incluyen aspectos sociales, económicos y ambientales.

El estado actual de conservación de la especie es el resultado de la conjugación de distintos factores antropogénicos y naturales que se han dado durante los últimos 450 años (Ruiz-Garduño et al., 2011), situación que debe de tomar en cuenta al momento de proponer y ejecutar acciones de conservaciones en la zona de pino azul, en Juchipila, Zacatecas. La gestión es uno de los apartados más importantes y críticos al momento de efectuar el manejo de áreas protegidas, es por eso 
que la expansión y fortalecimiento de las mismas trae consigo la exacerbación de conflictos y contradicciones entre los usuarios locales y los gestores externos (tomadores de decisiones, administradores, o los conservacionistas).

Es necesario iniciar un proceso de vinculación entre sociedad y ciencia (Hague et al., 2010), ya que esta interacción es en beneficio de los habitantes locales, trayendo a mediano y largo plazo resultados positivos para el territorio (Jones-Walters y Cil, 2011). Un mecanismo por el cual puede favorecerse este tipo de esquemas de gestión es por medio del pago por servicios ambientales en sus diferentes niveles de gobierno, desde el federal y estatal hasta el municipal; no obstante, se requiere el apoyo de personal y recursos económicos para implementar todas y cada una de las propuestas y que no queden en un documento o en una declaratoria.

Se resalta en esta sección, el trabajo realizado en otras regiones del planeta como lo es en Catalunya, España, específicamente, en la Reserva de la Biosfera del Montseny; en donde la producción de piñón comestible de la especie Pinus pinea (Linneo), es un ejemplo palpable de la labor y el desempeño de dueños y poseedores de predios con esta especie, han logrado posicionar el producto como uno de los más redituables económicamente, con un esquema de manejo y conservación dignos de ser mencionados y tomados como referente para la zona de pino azul en Zacatecas.

Es necesario que todos los interesados, habitantes y propietarios, tengan participación activa (Enqvist et al., 2014) en el manejo y gestión de la especie de pino azul en Zacatecas.

\section{Conclusiones}

La desvinculación con la sociedad directamente involucrada en los planes de conservación y manejo en las ANP’s del territorio nacional, específicamente en la zona de distribución natural de pino azul (Pinus maximartinezii) en Zacatecas, ha dado como resultado estrategias de conservación carentes de consolidación, lo que está directamente relacionado con una forma de imposición de reglas. En este orden, se entiende que han sido acciones no consensadas con los dueños de predios con presencia silvestre de pino azul en Zacatecas.

En este sentido, el presente trabajo contribuye con estrategias bien definidas en cada una de las líneas de acción propuestas por CONANP (secciones 3.1 y 3.2), lo que facilita un esquema de 
conservación del recurso natural, de una forma endógena, en donde el actor principal son los propietarios de pino azul en Zacatecas.

Sin embargo, es prioritario el crear mecanismos que faciliten a los propietarios de predios con pino azul en Zacatecas el que tengan rutas definidas que les permitan mejorar su participación y comprometerse de mejor manera en la protección de la especie y de su hábitat, además de participar más activamente en la rehabilitación de las áreas e intercambiar e incrementar el conocimiento de su entorno.

Se pudo constatar que la actitud de los propietarios de predios con pino azul en Zacatecas es favorable; esto en un sentido de tener una apertura a colaborar y ampliar su participación en actividades de conservación, manejo y aprovechamiento de recursos naturales. Sin embargo, denotan una inseguridad y desorientación en temas relacionados con protección, manejo, restauración, gestión y uso potencial de los recursos naturales en los que se desarrollan comúnmente, lo cual puede remediarse mediante capacitación.

Las especies presentes y en general el ecosistema de pino azul en Zacatecas tienen un vasto potencial para ser considerada como insignia en la conservación no sólo en el estado de Zacatecas, sino en todo el país, puesto que de inmediato ofrece empatía con los interesados por la conservación, además de que, al ser estudiado, pone de manifiesto una serie de interacciones bióticas poco descritas.

Sin duda es un espacio que favorecería la investigación científica aunado a la conservación, sin dejar pasar inadvertidos aspectos relacionados con desarrollo sustentable desde las comunidades aledañas, y específicamente de los propietarios de predios con pino azul en Zacatecas.

Es muy importante implementar las acciones antes señaladas, pues del 18 al 28 de mayo del año 2017 ocurrió un incendio forestal dentro del ecosistema del Pino Azul, el cual afectó a un $70 \%$ de la población de esta especie existente en la zona. La recuperación y protección de esta zona depende de la implementación adecuada y oportuna de planes y programas adecuados, que mejoren la gestión silvícola y la protección ambiental de los predios con Pino Azul. 


\section{Literatura citada}

Casas Anguita, J.; J. R. Repullo Labrador, y J. Donado Campos. 2003. La encuesta como técnica de investigación. Elaboración de cuestionarios y tratamiento estadístico de los datos (I). Aten Primaria 31 (8): 527-38.

Aurora-Jonsson, S. 2011. Virtue and vulnerability: Discourses on women, gender and climate change. Global Environmental Change 21 (2): 744-751.

Beschta, R. L. y W. J. Ripple. 2010. Mexican wolves, elk, and aspen in Arizona: Is there a trophic cascade?. Forest Ecology and Management 260 (5): 915-922.

Boada, M., J. Puig y C. Barriocanal. 2013. The Effects of Isolation and natural park coverage for landrace In situ conservation: An approach from the Montseny Mountains (NE Spain). Sustainability 5(2): 654-663.

Braverman I. 2014. Conservation without nature: the trouble with in situ versus ex situ conservation. Geoforum 51: 47-57.

Breck, S. W., B. M. Kluever, M. Panasci, J. Oakleaf, T. Johnson, W. Ballard, L. Howery y D. L. Bergman. 2011. Domestic calf mortality and producer detection rates in the Mexican wolf recovery area: Implications for livestock management and carnivore compensation schemes. Biological Conservation 144 (2): 930-936.

Brightsmith, D. J., A. Stronza y K. Holle. 2008. Ecotourism, conservation biology, and volunteer tourism: A mutually beneficial triumvirate. Biological Conservation 141 (11): 2832-2842.

Budowski, G. 1976. Tourism and Environmental Conservation: Conflict, Coexistence or Symbiosis?. Environmental Conservation 3 (1): 27-31.

Buta, N., S. M. Holland y K. Kaplanidou. 2014. Local communities and protected areas: The mediating role of place attachment for pro-environmental civic engagement. Journal of Outdoor Recreation and Tourism (5-6): 1-10.

Cámara de Diputados del H. Congreso de la Unión. 2014. LGEEPA (Ley General del Equilibrio Ecológico y Protección al Ambiente), 2014. Secretaría General, Secretaría de Servicios Parlamentarios. Promulgada en el Diario Oficial de la Federación el 28 de enero de 1988 y la última reforma DOF 16-01-2014.

Camargo Ponce de León, G. 2007. Manual básico de restauración ecológica participativa. Parques 
Nacionales Naturales de Colombia. Papel y Plástico Impresores Ltda. Bogotá, Colombia. 60 pp.

Carbonell, M. 2011. La reforma penal que México necesita. Consultado: 10/11/2014. En: http:// estepais.com/site/2011/la-reforma-penal-que-mexico-necesita/

Comisión Nacional de Áreas Protegidas (CONANP). 2014. Objetivos estratégicos de la CONANP. Consultado: 11/11/2014. En:

www.conanp.gob.mx/quienes_somos/objetivos_estrategicos.php

Diario Oficial de la Federación (DOF). 1949. Decreto de las Zonas protectoras y de repoblación las cuencas de alimentación de las obras de irrigación de los distritos nacionales de riego. Órgano del Gobierno Constitucional de los Estados Unidos Mexicanos. Primera sección. Tomo CLXXV. Núm. 29, del miércoles 3 de agosto.

Enqvist, J., M. Tengö y O. Bodin. 2014. Citizen networks in the Garden City: Protecting urban ecosystems in rapid urbanization. Landscape and Urban Planning 130: 24-35.

Ericson, J. A. 2006. A participatory approach to conservation in the Calakmul Biosphere Reserve, Campeche, Mexico. Landscape and Urban Planning 74: 242-266.

Farjon, A. 2013. Pinus maximartinezii. Consultado: 13/11/2014.En: www.iucnredlist.org

Fischer, A., L. C. Naiman, A. Lowassa, D. Randall y D. Rentsch. 2014. Explanatory factors for household involvement in illegal bushmeat hunting around Serengeti, Tanzania. Journal for Nature Conservation 22 (6): 491-496.

García-Zamora, R. 2005. Migración internacional y remesas colectivas en Zacatecas. Foreign Affairs 5 (3): 43-52. Edición para Iberoamérica en español.

Giannecchini, J. 1993. Ecotourism: New partners, new relationships. Conservation Biology 7 (2): 429-432.

González-Elizondo, M., M. S. González-Elizondo, L. Ruacho-González y M. Molina-Olvera. 2011. Pinus maximartinezii (Rzed.) (Pinaceae). Primer registro para Durango, segunda localidad para la especie. Acta Botánica Mexicana 96: 33-48.

González-Oliva, L., Z. Acosta Ramos y A. J. Urquiola Cruz. 2011. Translocación y cultivo ex situ de Amaranthus minimus, una hierba endémica y amenazada. Revista del Jardín Botánico Nacional 32/33: 169-172.

Groom, J. D., L. B. McKinney, L. C. Ball y C. S. Winchell. 2007. Quantifying off-highway vehicle 
impacts on density and survival of a threatened dune-endemic plant. Biological Conservation 135 (1): 119-134.

Hague, M., P. Leroy y A. C. Petersen. 2010. Stakeholder participation in environmental knowledge production. Futures 42 (3): 254-264.

Jones-Walters, L. y Aysegül Cil. (2011). Biodiversity and stakeholder participation. Journal for Nature Conservation 19 (6): 327- 329.

Karanth, K. K. y R. DeFries. 2011. Nature-based tourism in Indian protected areas: New challenges for park management. Conservation Letters 4 (2): 137-149.

Karki, S. T. 2013. Do protected areas and conservation incentives contribute to sustainable livelihoods? A case study of Bardia National Park, Nepal. Journal of Environmental Management 128: 988-999.

López-Espinosa De los Monteros, R. 2002. Evaluating ecotourism in natural protected areas of La Paz Bay, Baja California Sur, Mexico: Ecotourism or nature-based tourism? Biodiversity and Conservation 11, 1539-1550.

López-Mata, L. e I. G. Galván Escobedo. 2011. Extracción de semillas de Pinus maximartinezii y sus consecuencias poblacionales. Biodiversitas 98: 1-7.

López-Mata, L. 2013. The Impact of Seed Extraction on the population dynamics of Pinus maximartinezii. Acta Oecologica 49: 39-44.

Luque Gil, A. M. 2004. Evaluación de la aptitud del territorio para la práctica del senderismo. Servicio de Publicaciones y Divulgación Científica de la Universidad de Málaga. Málaga, España. $129 \mathrm{pp}$.

Méndez-López M. E., E. García-Frapolli, D. J. Pritchard, M. C. Sánchez González, I. Ruiz-Mallén, L. Porter-Bolland y V. Reyes-García. 2014. Local participation in biodiversity conservation initiatives: A comparative analysis of different models in South East México. Journal of Environmental Management 145: 321-329.

Meinzen-Dick, R., L. R. Brown, H. S. Feldstein y A. Quisumbing. 1997. Gender, property rights, and natural resources. World Development 25 (8): 1303-1315.

Mittermeier, R. A. y M. C. Mittermeier. 1992. La importancia de la diversidad biológica en México. pp. 63-73. En: Sarukhán, J. y R. Dirzo (Eds.). México ante los retos de la Biodiversidad. CONABIO. Ciudad de México, México. 343 pp.

Ocaña Ocaña, M. C., M. L. Gómez Moreno, J. A. Arrebola Castaño, R. Blanco Sepúlveda, F. B. 
Galacho Jiménez, R. Larrubia Vargas, A. M. Luque Gil, M. F. Mérida Rodríguez, S. R. Navarro Rodríguez, L. M. Rubio Barquero y J. M. Vías Martínez. 2012. Desarrollo metodológico sobre la evaluación de la capacidad de uso recreativo de espacios protegidos. Nimbus: Revista de Climatología, Meteorología y Paisaje 29-30: 447-460.

Ocaña Ocaña, M. C., R. Larrubia Vargas y S. R. Navarro Rodríguez. 2013. Incidencia del senderismo en la economía local. Un análisis empírico a propósito del Parque Natural Sierra de las Nieves (Málaga). Anales de Geografía de la Universidad Complutense 33 (1): 161-192.

O'Shaughnessy, S. y N. T. Krogman. 2011. Gender as contradiction: From dichotomies to diversity in natural resource extraction. Journal of Rural Studies 27 (2): 134-143.

Otero, L., M. Boada, A. Badia, E. Pla, J. Vayreda, S. Sabaté, C. A. Gracia y J. Peñuelas. 2011. Loss of water availability and stream biodiversity under land abandonment and climate change in a Mediterranean catchment (Olzinelles, NE Spain). Land Use Policy 28 (1): 207-218.

Padilla, J. M. 2006. Cambio sociodemográfico y económico de la región Juchipila, Zacatecas. Revista Digital de la Universidad Autónoma de Zacatecas de Investigación Científica 2 (2): 1-24.

Pietrzyk-Kaszynska, A., J. Cent, M. Grodzinska-Jurczaka y M. Szymanska. 2012. Factors influencing perception of protected areas - The case of Natura 2000 in Polish Carpathian communities. Journal for Nature Conservation, 20 (5): 284-292.

Procuraduría Federal de Protección al Ambiente (PROFEPA). 2014. Misión, Visión y Objetivos Estratégicos de la PROFEPA. Consultado: 15/11/2014. En: www.profepa.gob.mx/ innovaportal/v/1168/1/mx/vision_mision_y_objetivos_estrategicos.html

Programa de Acción para la Conservación de las Especies (PACE). 2007. Cotorras Serranas (Rhynchopsitta spp). SEMARNAT (Secretaria de Medio Ambiente y Recursos Naturales). Ciudad de México, México.58 pp.

Rodríguez-Martínez, R.E. 2007. Community involvement in marine protected areas: The case of Puerto Morelos reef, México. Journal of Environmental Management 88 (4): 1151-1160.

Ruiz Garduño, R. R., M. Márquez Madrid, R.D. Valdez Cepeda, F. Blanco Macías y J. C. Ledesma Mares. 2011. Estado y conservación del pino azul (Pinus maximartinezii) y sus especies asociadas en la Sierra de Juchipila, Zacatecas. pp. 351-361. En: Laclette J.P, P. Zúñiga y J. A. Romero (Eds.). El Impacto de los Fondos Mixtos en el Desarrollo Regional. CONACYT, FCCYT y REDNACECYT. Ciudad de México, México. 390 pp. 
Sanyé-Mengual, E., H. Romanos, C. Molina, M. Antonia Oliver, N. Ruiz, M. Pérez, D. Carreras, M. Boada, J. Garcia-Orellana, J. Duch y J. Rieradeval. 2014. Environmental and self-sufficiency assessment of the energy metabolism of tourist hubs on Mediterranean Islands: The case of Menorca (Spain). Energy Policy 65: 377-387.

Secretaría de Medio Ambiente y Recursos Naturales (SEMARNAT). 2010. Norma Oficial Mexicana NOM-059-ECOL-2010, Protección ambiental-Especies Nativas de México de Flora y Fauna Silvestres-Categorías de Riesgo y Especificaciones para su Inclusión, Exclusión o cambioLista de Especies en Riesgo. Diario Oficial de la Federación, Segunda Sección, jueves 30 de diciembre de 2010.

Secretaría de Medio Ambiente y Recursos Naturales (SEMARNAT). 2010. Biodiversidad, conocer para conservar. Serie ¿y el Medio Ambiente? Secretaría de Medio Ambiente y Recursos Naturales. Ciudad de México, México. 210 pp.

Sierra-Bravo, R. 1994. Técnicas de Investigación Social. Editorial Paraninfo SA. Madrid, España. 349 pp.

Soulé, M. E. 1987. News of the society. Conservation Biology 1 (1): 4-5.

Stronza, A. 2007. The economic promise of ecotourism for conservation. Journal of Ecotourism 6 (3): 210-230.

Tole, L. 2010. Reforms from the Ground up: A review of community-based forest management in tropical developing countries. Environmental Management 45: 1312-1331.

Toledo, V.M. 2005. Repensar la conservación: ¿áreas naturales protegidas o estrategia bioregional? Gaceta Ecológica 77: 67-83.

Turbay Ceballos, S., C. Maldonado Lizarazo, E. Montalvo, H. Velásquez y J. C. Perdomo 2013a. Lecciones de una experiencia de participación campesina en un proceso de restauración ecológica en el Parque Nacional Natural Las Orquídeas, Colombia. Gestión y Ambiente 16 (1): 5-15.

Wolcott, T. G. y D. L. Wolcott. 1984. Impact of off-road vehicles on macroinvertebrates of a mid-atlantic beach. Biological Conservation 29 (3): 217-240.

Zhou, Y., C. D. Buesching, C. Newman, Y. Kaneko, Z. Xie y D. W. Macdonald. 2013. Balancing the benefits of ecotourism and development: The effects of visitor trail-use on mammals in a Protected Area in rapidly developing China. Biological Conservation 165: 18-24. 


\section{Cita:}

Hernández-Ramírez, D., E. Alanís-Rodríguez, M. Boada, C. Barriocanal, R. Maneja y Jorge BluhmGutiérrez. 2018. Elementos para la gestión de la conservación, manejo y aprovechamiento del pino azul (Pinus maximartinezii Rzed.) en Juchipila, Zacatecas, México. 2018. Vol. 4 (1): 73-95. https:// doi.org/10.18242/anpscripta.2018.04.04.01.0004

Sometido: 14 de Octubre de 2017

Revisado: 17 de Diciembre de 2017

Aceptado: 19 de Marzo de 2018

Editora asociada: Dra. Cecilia Jiménez Sierra

Idioma Inglés Abstract: Ms.C. Diana Dorantes

Diseño gráfico editorial: Lic. Gerardo Hernández 